\title{
ESTRUTURA DA TECNOLOGIA DE PRODUÇÃO AGRICOLA REGIONAL: UMA ABORDAGEM DA PROGRAMAÇÃO DE METAS ${ }^{1}$
}

\author{
Antonio José Medina dos Santos Baptista ${ }^{2}$ \\ William Romell Benevides de Ávila \\ João Eustáquio de Lima ${ }^{4}$ \\ Adriano Provezano Gomes 5
}

\begin{abstract}
Resumo - Este estudo objetivou estimar e analisar as funções de produção média e de fronteira e caracterizar a estrutura da tecnologia de produção agrícola. Com base nos dados do Censo Agropecuário de 1995/96, referente à mesorregião do Triângulo Mineiro e Alto Paranaíba do Estado de Minas Gerais, utilizou-se a programação de metas (goal programming) para calcular os parâmetros das funções de produção na forma funcional tipo Cobb Douglas. Uma das principais conclusões é o fato de que a abordagem utilizada no estudo permite identificar as significativas diferenças no nível de eficiência produtiva e na estrutura das tecnologias de produção na região, indicando o viés que se incorre ao tomar decisões baseadas somente nos parâmetros da função de produção média. Outra conclusão importante é o fato de que os procedimentos adotados no estudo conseguem evidenciar algumas características estruturais da tecnologia de produção, oferecendo maiores informações sobre a tomada de decisão.
\end{abstract}

Palavras-chave: Função de produção, programação de metas, eficiência e agropecuária.

\footnotetext{
Recebido em 21/07/2005 Aceito em 01/12/2005.

Professor da Universidade Jean Piaget de Cabo Verde. E-mail: tozecv@yahoo.com.br

Mestre em Economia Aplicada pela Universidade Federal de Viçosa. E-mail: wravila@bol.com.br

4 Professor do Departamento de Economia Rural da Universidade Federal de Viçosa. E-mail: jelima@ufv.br

5 Professor do Departamento de Economia da Universidade Federal de Viçosa. E-mail: apgomes@ufv.br
} 


\section{Introdução}

A agropecuária ocupa posição de destaque na economia nacional. Além do grande contingente de mão-de-obra que emprega, participa na segurança alimentar e gera divisas que poderão ser alocadas noutros setores da economia e impulsionar o desenvolvimento econômico do país.

Como a agropecuária é um dos setores importantes dentro da estratégia de desenvolvimento e segurança alimentar, é imprescindível que sejam realizados estudos que subsidiem o governo e demais tomadores de decisão na elaboração de estratégias de ação que possam facilitar a participação da agropecuária, de forma efetiva, no processo de desenvolvimento econômico brasileiro.

A função de produção é um importante instrumento de análise do setor agrícola, e sua estimação e interpretação, de forma correta, podem facilitar a tomada de decisão e melhorar, significativamente, a alocação dos escassos recursos do governo e setor privado. No intuito de tornar mais coerentes as políticas adotadas pelo setor agrícola, é importante disponibilizar novos procedimentos empíricos de análise da função de produção, os quais permitirão tomadas de decisão mais coerentes e com maior suporte.

Neste sentido, este trabalho objetivou buscar uma alternativa de estimação dos parâmetros da função de produção, com vistas em caracterizar, mais detalhadamente, a estrutura da tecnologia de produção agropecuária e evidenciar a distribuição da ineficiência produtiva das unidades de produção analisadas. Para isso, serão utilizados dados do Censo Agropecuário para os municípios da mesorregião Triângulo Mineiro/Alto Paranaíba, localizada no Estado de Minas Gerais. 
Antonio José Medina dos Santos Baptista, William Romell Benevídes de Ávila, João Eustáquio de Lima \& Adriano Provezano Gomes

\section{Metodologia}

A tecnologia da produção agropecuária, geralmente, é representada pelas funções de produção. Por meio dos parâmetros da função caracterizamse as diferentes estruturas das tecnologias de produção.

Segundo Debertin (1986), de forma genérica, uma função de produção pode ser representada, algebricamente, por

$\mathrm{Y}=\mathrm{f}\left(\mathrm{X}_{1}, \mathrm{X}_{2}, \ldots, \mathrm{X}_{\mathrm{n}}\right)$

em que $Y$ é a variável dependente e indica a quantidade produzida por unidade de tempo, e $X_{i,} i=1,2, \ldots, n$, variáveis independentes que representam os fatores utilizados na produção. A função de produção $(f)$ indica a relação técnica entre a produção máxima obtida em determinada unidade de tempo e os fatores utilizados no processo de produção.

A simples agregação das unidades de produção e a subseqüente estimação econométrica geram resultados viesados que podem comprometer a alocação eficiente dos recursos, que atualmente são escassos e caros. De acordo com Sato (1975), a agregação das microfunções de produção para obter uma macrofunção de produção, sem levar em conta as diferenças na eficiência produtiva, gera resultados viesados, razão por que na estimação da função de produção agregada devem-se levar em conta as características de eficiência produtiva das diferentes unidades.

Incorre em erros ao tentar comparar diferentes unidades de produção com base apenas na estimação de função de produção média, pelo fato de que esta não leva em consideração as diferenças na eficiência produtiva entre as unidades de produção. Com isso, podem-se gerar estimativas de parâmetros que não caracterizam a estrutura da produção de nenhum grupo relevante de unidades (ex: caso haja dois grandes agrupamentos de unidades de produção, um próximo da fronteira eficiente e outro próximo da fronteira ineficiente, a função média estimada passará entre 
os dois grandes grupos, sem representar nenhuma das tecnologias predominantes na amostra analisada).

Geralmente, de acordo com Fare et al. (1994), a estimação de funções de produção pode ser realizada por meio de abordagem paramétrica (econométrica) ou não-paramétrica (programação matemática). A maioria dos estudos que analisam a função de produção utiliza a média estimada por técnicas econométricas. Entretanto, como citado anteriormente, este procedimento pode levar a resultados viesados, pelo fato de não levar em consideração as diferenças na eficiência produtiva. Além disso, não fornece um intervalo de variação dos parâmetros estimados, isto é, a estimação da função média gera o valor dos parâmetros da função que se verifica na média, sem nenhuma evidência da função relevante que caracteriza a tecnologia ineficiente e a eficiente. Neste sentido, torna-se necessário estimar não só a função média, como também uma função de produção de fronteira que caracterize a melhor tecnologia e a função de produção relevante para os ineficientes. A estimação dessas funções permitirá fazer comparações entre as unidades de produção em termos de eficiência produtiva, além de oferecer uma caracterização mais detalhada da estrutura da tecnologia de produção relevante na amostra analisada.

Para determinar melhor as diferenças entre as funções de produção, a Figura 1 ilustra uma função de produção média, estimada por mínimos quadrados, e as funções de produção de fronteira eficiente e ineficiente. 


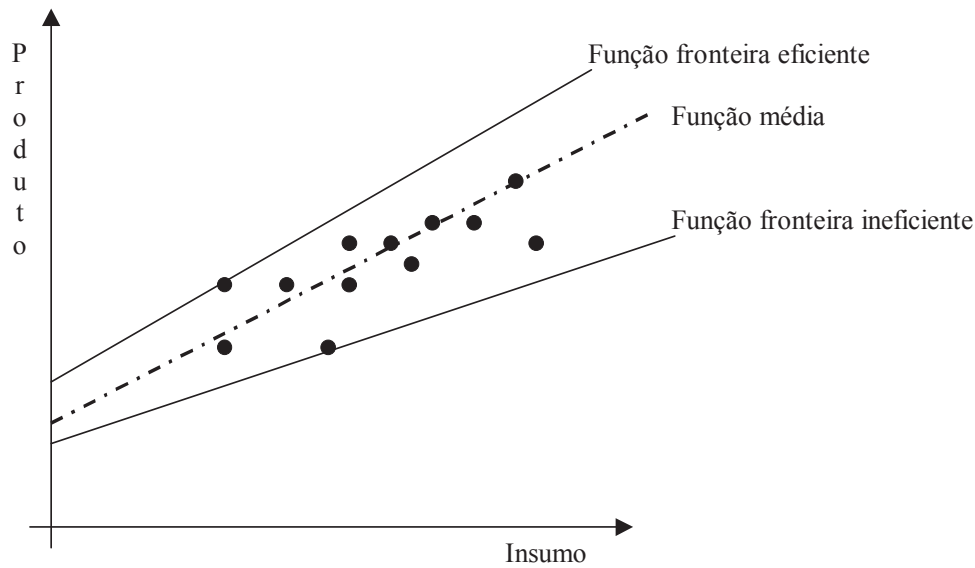

Figura 1- Representação das funções de produção média e de fronteira.

Como se pode observar, na função média, ao minimizar o quadrado dos desvios, os resíduos passam a ter valores tanto positivos como negativos. Já na função fronteira eficiente, todos os resíduos serão zero (unidades eficientes) ou negativos, enquanto na função de fronteira ineficiente, todos serão iguais a zero (ineficientes) ou positivos.

A existência de ineficiências dificulta a estimação precisa dos parâmetros da função de produção, enviesando as estimativas, de acordo com a distribuição da ineficiência das unidades de produção ${ }^{6}$. Em outras palavras, se a produção for dominada por unidades ineficientes, os parâmetros da função média serão semelhantes aos da função que caracteriza as unidades ineficientes. Situação contrária será verificada, caso o número de unidades eficientes domine, ou seja, os parâmetros da função média tenderão a se assemelhar aos da função eficiente.

O viés que se refere no texto não deve ser confundido com o viés do estimador (O MQO é "blue"), pois referese à possibilidade de existir diferentes valores dos parâmetros, dependendo da distribuição da ineficiência na amostra. 
Na estimação empírica da função de produção utilizou-se a forma funcional tipo Cobb Douglas. Optou-se por esta forma funcional, para representar a tecnologia da produção, por ser ela parcimoniosa em relação à quantidade de parâmetros; pela facilidade de interpretação dos parâmetros; por ser linearizada por meio de logaritmos; e por ter uso generalizado nas análises empíricas da produção agrícola.

A função tipo Cobb Douglas, com três fatores de produção, pode ser representada, algebricamente, por

$$
\mathrm{Y}=\mathrm{AX}_{1}^{\beta_{1}} \mathrm{X}_{2}^{\beta_{2}} \mathrm{X}_{3}^{\beta_{3}}
$$

Ao aplicar logaritmos em ambos os lados da equação (2) e acrescentar o termo de erro, tem-se

$$
\ln Y_{i}=\alpha+\beta_{1} \ln X_{1 i}+\beta_{2} \ln X_{2 i}+\beta_{3} \ln X_{3 i}+\varepsilon_{i}
$$

em que $\ln Y_{\mathrm{i}}$ é o logaritmo da variável dependente e indica a quantidade produzida pela i-ésima unidade de produção, $\ln \mathrm{X}_{\mathrm{ji}}$, variáveis independentes, que representam o j-ésimo fator de produção utilizado pela i-ésima unidade de produção; $\varepsilon$, resíduo estocástico, que se pressupõe ter média zero e variância constante; os coeficientes $\alpha$ e $\beta_{\mathrm{j}}$ são parâmetros a serem estimados, sendo $\beta_{\mathrm{j}}$ as elasticidades de produção do j-ésimo fator de produção, e $\alpha=\ln \mathrm{A}$.

A estimação das funções de produção será feita pela Programação Quadrática (PPQ), no âmbito da Programação de Metas (Goal Programming), em que se pretende selecionar os valores dos parâmetros da função, de modo que a soma dos quadrados dos resíduos seja mínima. 
Antonio José Medina dos Santos Baptista, William Romell Benevídes de Ávila,

João Eustáquio de Lima \& Adriano Provezano Gomes

Neste estudo utilizou-se a abordagem proposta por Aigner e Chu (1968), para estimar as funções de fronteira. Serão modelados três Problemas de $\mathrm{PPQ}^{7}$, cujas funções consistem em Minimizar a Soma dos Quadrados dos Resíduos, diferenciando-se apenas nas pressuposições quanto à distribuição dos resíduos. A função média será estimada por MQO (Mínimos Quadrados Ordinários), a qual pode ser considerada um PPQ sem as restrições nos resíduos.

Para estimar as funções de produção fronteira eficiente e ineficiente deve-se incorporar, a priori, a pressuposição de que os resíduos tenham valores negativos ou positivos, respectivamente, podendo ser representados por meio dos seguintes Problemas de Programação Quadrática.

Para a fronteira eficiente:

$\operatorname{Min}_{\alpha, \beta} \sum \varepsilon_{\mathrm{i}}^{2}=\operatorname{Min}_{\alpha, \beta} \sum\left(\ln \mathrm{Y}_{\mathrm{i}}-\left(\alpha+\beta_{1} \ln \mathrm{X}_{1 \mathrm{i}}+\beta_{2} \ln \mathrm{X}_{2 \mathrm{i}}+\beta_{3} \ln \mathrm{X}_{3 \mathrm{i}}\right)\right)^{2}$

S.a.

$\varepsilon_{\mathrm{i}} \leq 0 \therefore \ln \mathrm{Y}_{\mathrm{i}}-\left(\alpha+\beta_{1} \ln \mathrm{X}_{1 \mathrm{i}}+\beta_{2} \ln \mathrm{X}_{2 \mathrm{i}}+\beta_{3} \ln \mathrm{X}_{3 \mathrm{i}}\right) \leq 0$.

Para a fronteira ineficiente:

$\underset{\alpha, \beta}{\operatorname{Min}} \sum \varepsilon_{\mathrm{i}}^{2}=\underset{\alpha, \beta}{\operatorname{Min}} \sum\left(\ln \mathrm{Y}_{\mathrm{i}}-\left(\alpha+\beta_{1} \ln \mathrm{X}_{1 \mathrm{i}}+\beta_{2} \ln \mathrm{X}_{2 \mathrm{i}}+\beta_{3} \ln \mathrm{X}_{3 \mathrm{i}}\right)\right)^{2}$

S.a.

$\varepsilon_{\mathrm{i}} \geq 0 \therefore \ln \mathrm{Y}_{\mathrm{i}}-\left(\alpha+\beta_{1} \ln \mathrm{X}_{1 \mathrm{i}}+\beta_{2} \ln \mathrm{X}_{2 \mathrm{i}}+\beta_{3} \ln \mathrm{X}_{3 \mathrm{i}}\right) \geq 0$.

\footnotetext{
A proposta original de Aigner e Chu (1968) contempla apenas a estimação da fronteira eficiente. Neste estudo estimou-se também a fronteira ineficiente e a média, para fazer comparações na estrutura da tecnologia da produção dos diferentes grupos de eficiência.
} 
De acordo com Schmidt (1976) e Kunbhakar e Lovell (2000), a estimação dos parâmetros por meio dos Problemas de Programação Quadrática, apresentados em (4) e (5), gera resultados semelhantes à estimação das funções fronteiras determinísticas por máxima verossimilhança, quando há pressuposição de que os resíduos tenham distribuição half-normal.

Como se pode observar, pelos modelos apresentados em (4) e (5), apenas se calculam os valores dos parâmetros, e não o erro-padrão, que possibilitam fazer inferências estatísticas sobre os parâmetros. Outra limitação do modelo utilizado é a sensibilidade a observações discrepantes (outliers), que podem comprometer, de forma significativa, a estimação das fronteiras.

Após estimar os parâmetros do PPQ apresentado em (4), podem-se estimar escores de eficiência das unidades de produção, por meio da seguinte expressão:

$$
\mathrm{ET}_{\mathrm{i}}=\mathrm{e}^{\hat{\varepsilon}_{\mathrm{i}}}
$$

em que $\mathrm{ET}_{\mathrm{i}}$ é o escorre de eficiência técnica da i-ésima unidade de produção, com valor entre 0 e 1 ; quanto mais próximo de 1 , maior será o nível de eficiência da unidade de produção; “e”, exponencial; e $\hat{\varepsilon}_{\mathrm{i}}$, resíduo estimado para a i-ésima unidade de produção, calculado pelo PPQ apresentado em (4).

Após obter os coeficientes das funções de produção média, eficiente e ineficiente, podem-se analisar as diferenças nas tecnologias utilizadas pelos diferentes grupos de unidades de produção, assim como o nível de eficiência técnica destas. Ao observar as relações entre os diferentes parâmetros $\beta$ estimados de cada função, pode-se ter idéia da dispersão das elasticidades de produção entre as unidades de produção, isto é, se os parâmetros das elasticidades de produção estimados para a média são semelhantes aos das unidades ineficientes ou das eficientes. Dessa 
forma, pode-se caracterizar a tecnologia da produção de forma mais extensiva, analisando as relações

$\mathbf{A}=\frac{\beta_{\text {médio }}}{\beta_{\text {eficientes }}}$,
$\mathbf{B}=\frac{\beta_{\text {médio }}}{\beta_{\text {ineficientes }}}$,

em que $\beta_{\text {medio }}$ é o j-éssimo parâmetro estimado da função de produção média; $\beta_{\text {eficiente }}$ j-éssimo parâmetro estimado da função de produção de fronteira eficiente; e $\beta_{\text {ineficiente }}$, j-éssimo parâmetro estimado da função de produção de fronteira ineficiente. Ao verificar a relação A, nota-se que quanto mais próxima da unidade, maior semelhança terá a média com as unidades eficientes de produção; no entanto, quanto mais próxima da unidade for a relação $\mathrm{B}$, maior proximidade terá da média com as unidades ineficientes. Essas informações são importantes para identificar os fatores de produção que não estão sendo utilizados, de forma eficiente, na região sob análise.

\subsection{Região de estudo e dados utilizados}

Para ilustrar a aplicação dos procedimentos analíticos propostos, foram utilizados dados coletados no Censo Agropecuário de 1995/96 $6^{8}$, referentes aos 64 municípios da mesorregião Triângulo Mineiro/Alto Paranaíba do Estado de Minas Gerais. A escolha desta mesorregião está na sua importância relativa no estado de Minas Gerais, como se pode observar nos dados da Tabela 1.

\footnotetext{
8 As informações sobre insumos e produto agrícolas municipais somente estão disponíveis no Censo Agropecuário, cuja última edição ocorreu em 1995/96.
} 
Tabela 1 - Distribuição percentual da produção de leite e do valor da produção agrícola no estado de Minas Gerais, no ano de 2003

\begin{tabular}{lccc}
\hline & \multicolumn{3}{c}{ Distribuição (\%) } \\
\cline { 2 - 4 } Mesorregião & Leite & $\begin{array}{c}\text { Lavoura } \\
\text { temporária }\end{array}$ & $\begin{array}{c}\text { Lavoura } \\
\text { permanente }\end{array}$ \\
\hline Noroeste de Minas & 5,07 & 17,67 & 1,66 \\
Norte de Minas & 3,70 & 5,20 & 5,29 \\
Jequitinhonha & 2,18 & 1,50 & 2,44 \\
Vale do Mucuri & 2,46 & 0,54 & 1,08 \\
Triângulo Mineiro/Alto Paranaíba & $\mathbf{2 4 , 8 3}$ & $\mathbf{4 2 , 3 4}$ & $\mathbf{2 3 , 9 3}$ \\
Central Mineira & 8,53 & 2,23 & 0,11 \\
Metropolitana de Belo Horizonte & 7,91 & 2,77 & 1,66 \\
Vale do Rio Doce & 6,88 & 3,00 & 6,07 \\
Oeste de Minas & 8,59 & 4,29 & 100,00 \\
Sul/Sudoeste de Minas & 100,00 & 100,00 & 6,47 \\
Campo das Vertentes & 15,85 & 14,87 & 33,38 \\
Zona da Mata & 4,46 & 2,15 & 2,76 \\
\hline & & 3,46 & 15 \\
Minas Gerais & & & \\
\hline
\end{tabular}

Fonte: IBGE (2005) - Pesquisa da Pecuária Municipal e Produção Agrícola Municipal. 
Antonio José Medina dos Santos Baptista, William Romell Benevídes de Ávila, João Eustáquio de Lima \& Adriano Provezano Gomes

Como se pode verificar, a mesorregião Triângulo Mineiro/Alto Paranaíba ocupa a primeira colocação na produção de leite e no valor da produção de lavouras temporárias no estado, além de ser a segunda colocada no ranking do valor da produção de lavouras permanentes em Minas Gerais.

Essa região, típica de cerrado, destacou-se nos últimos anos como a principal produtora de grãos e pecuária, tanto de corte quanto de leite. A produção vegetal, nessa região, foi beneficiada pelas tecnologias desenvolvidas em diversos centros de pesquisa, as quais buscaram viabilizar a produção agrícola no cerrado brasileiro. Nessa região mineira, há muita semelhança com a agricultura desenvolvida no Centro-Oeste brasileiro, com destaque para o estado de Goiás, maior celeiro de produção agrícola nacional.

Em relação à pecuária, essa região sempre se destacou na produção de carne, em virtude, principalmente, da topografia plana. Entretanto, nas últimas décadas, as regiões tradicionais na produção de leite, como Sul de Minas e Zona da Mata, estão perdendo espaço para a região do Triângulo Mineiro/Alto Paranaíba. Ressalta-se que Minas Gerais é responsável por cerca de $1 / 3$ da produção de leite no Brasil, e, deste total, $25 \%$ ocorre na mesorregião Triângulo Mineiro/Alto Paranaíba.

Para estimar as funções de produção, foram utilizadas as seguintes variáveis:

- Terra $\left(X_{1}\right)$ : área explorada, expressa em mil hectares. O conceito de área explorada refere-se à soma de áreas com lavouras permanentes e temporárias, pastagens plantadas, matas plantadas, áreas com pastagens naturais e matas naturais.

- Despesas $\left(\mathrm{X}_{2}\right)$ : expressa em mil reais de 1996, agrega o total de gastos realizados no processo produtivo. Essa variável pode ser interpretada como proxy de capital, pelo fato de envolver gastos com combustíveis e lubrificantes, adubos e corretivos, medicamentos para animais, controles de pragas e doenças, ração e outros itens da alimentação animal. 
- Trabalho $\left(\mathrm{X}_{3}\right)$ : pessoal ocupado nas atividades agropecuárias, expresso em número de trabalhadores.

- Produção (Y): soma do valor da produção vegetal e animal, expressas em mil Reais de 1996.

\section{Resultados e discussões}

$\mathrm{Na}$ Tabela 2, encontram-se os parâmetros das diferentes funções de produção estimadas. Como se pode observar, a função objetivo (Min $\Sigma e^{2}$ - Minimizar a Soma dos Quadrados dos Resíduos) obteve o melhor (menor) valor na função média, como era de esperar, sendo o pior valor (maior) verificado na função fronteira ineficiente. O parâmetro a, que geralmente é interpretado nas funções de produção tipo Cobb-Douglas como parâmetro de eficiência, apresentou certa coerência nos resultados, visto que seu valor para a fronteira eficiente foi maior do que os das demais funções de produção estimadas. Quanto aos demais parâmetros, nota-se que as elasticidades de produção da terra e da despesa, referentes à função fronteira eficiente, foram maiores do que as estimadas para as demais funções de produção, enquanto a fronteira ineficiente apresentou maior elasticidade de produção do fator trabalho.

Em relação à elasticidade de escala (retornos à escala), nota-se que os valores são próximos de um. Sem muita precisão, pode-se considerar que todas as funções estimadas apresentam retornos constantes à escala. 
Antonio José Medina dos Santos Baptista, William Romell Benevídes de Ávila,

João Eustáquio de Lima \& Adriano Provezano Gomes

Tabela 2 - Parâmetros das funções de produção estimadas por PPQ

\begin{tabular}{lccc}
\hline \multicolumn{1}{c}{ Parâmetro } & Fronteira Eficiente & Média & Fronteira Ineficiente \\
\hline $\mathrm{A}=e^{\alpha}$ & 1,2674 & 1,2444 & 0,6188 \\
$\beta_{1}$ (Terra) & 0,2090 & 0,1622 & 0,1986 \\
$\beta_{2}$ (Despesa) & 0,7343 & 0,7114 & 0,5703 \\
$\beta_{3}$ (Trabalho) & 0,0924 & 0,1473 & 0,2919 \\
Retornos à escala & 1,0357 & 1,0210 & 1,0609 \\
$\sum \hat{\varepsilon}_{i}^{2}$ & 9,1237 & 2,1629 & 16,8023 \\
\hline
\end{tabular}

Fonte: Dados da pesquisa.

Os parâmetros da função fronteira eficiente permitem analisar a produção potencial e calcular a distância de cada unidade de produção da fronteira de produção. Dessa forma, os resíduos (negativos) são indicadores de ineficiência técnica relativa à fronteira, isto é, indicam a falha das unidades de produção em alcançar a produção potencial.

Pela equação apresentada em (6) calcularam-se os escorres de eficiência para cada município da amostra, sendo os resultados apresentados por meio de histograma na Figura 2. Como se pode verificar, $67 \%$ do total de 64 municípios da amostra apresentaram escorres de eficiência entre 0,635 e 0,854 .

A média dos escorres de eficiência técnica, calculada para os municípios da amostra, foi de 0,73. Para se ter idéia da dispersão das observações em torno do valor médio, calculou-se o coeficiente de variação, cujo valor foi de $18,08 \%$, o que indica considerável variação das observações em torno da média e reforça a necessidade de uma análise individualizada. Outras estatísticas calculadas foram o desvio-padrão $(0,132)$, o escorre mínimo observado $(0,416)$ e o escorre máximo, que foi de 1 . 


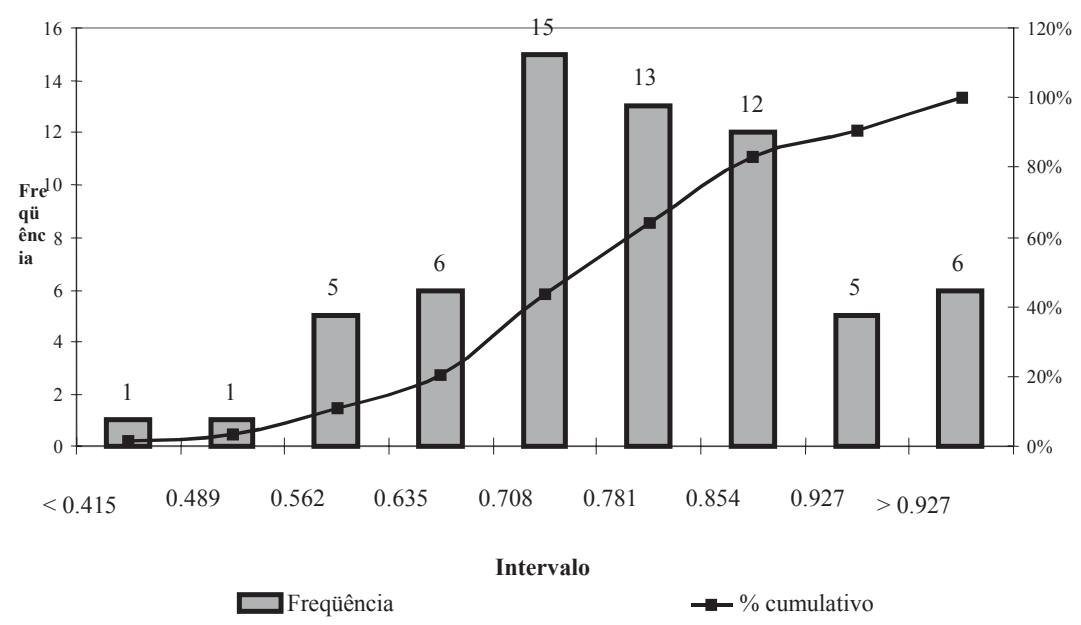

Figura 2 - Histograma de freqüência da distribuição da eficiência técnica na agropecuária da mesorregião de Triângulo Mineiro/Alto Paranaíba, 1995/96.

Fonte: Dados da pesquisa.

Pelos resultados da Tabela 3, pode-se ter idéia mais detalhada da estrutura da tecnologia na região analisada. Isso pode ser feito ao verificar as diferenças nos parâmetros das diferentes funções estimadas, as quais permitem inferir sobre a característica da tecnologia predominante na região.

Por meio das relações apresentadas em (7) e (8) e pelos resultados apresentados na Tabela 2, podem-se calcular as relações A e B, as quais caracterizam a tecnologia de produção. Exemplificando com o fator de produção terra, tem-se: 


$$
\begin{aligned}
& \mathbf{A}=\frac{\beta_{\text {médio }}}{\beta_{\text {eficientes }}}=\frac{0,1622}{0,2090}=0,7762, \\
& \mathbf{B}=\frac{\beta_{\text {médio }}}{\beta_{\text {ineficientes }}}=\frac{0,1622}{0,1986}=0,8167 .
\end{aligned}
$$

Nota-se que, na região utilizada no estudo, a maioria dos municípios apresenta elasticidades de produção do fator terra semelhante aos ineficientes, pelo fato de $\mathrm{B}(0,8167)$ ser mais próximo de 1 do que $\mathrm{A}$ $(0,7762)$. Repetindo o mesmo procedimento em relação aos fatores de produção despesa e do trabalho e em relação às elasticidades de escala, chega-se aos resultados apresentados na Tabela 3.

Tabela 3 - Caracterização da tecnologia da produção agrícola na mesorregião do Triângulo Mineiro/Alto Paranaíba, em 1995/96

\begin{tabular}{llll}
\hline Parâmetros & $\mathrm{A}$ & $\mathrm{B}$ & Semelhança \\
\hline$\beta$ (Terra) & 0,7762 & 0,8167 & Ineficiente \\
$\beta_{2}$ (Despesa) & 0,9688 & 1,2474 & Eficiente \\
$\beta_{3}$ (Trabalho) & 1,5946 & 0,5048 & Ineficiente \\
Retornos à escala & 0,9857 & 0,9624 & Eficiente \\
\hline
\end{tabular}

Fonte: Dados da pesquisa.

Em relação à elasticidade de produção da despesa e retornos à escala, percebe-se que a maioria dos municípios se assemelha aos eficientes. Já em relação à elasticidade de produção do fator trabalho, nota-se semelhança com os ineficientes. Cabe lembrar que a questão de semelhança utilizada na análise é verificada pelas relações A e B, ou seja, quanto mais próximo de 1 , mais semelhante será a média das 
unidades de produção das eficientes, caso a relação A seja mais próxima de 1 do que de $\mathrm{B}$; caso contrário, haverá semelhança com a fronteira ineficiente.

Dessa forma, podem-se atribuir prioridades na formulação de políticas agrícolas, para que se conheça a distribuição da eficiência técnica relativa na região, assim como as elasticidades de produção relevantes para a maioria das unidades de produção. No caso do presente estudo, nota-se que existem diferenças significativas no desempenho da produção agropecuária, o que faz com que as políticas de desenvolvimento sejam diferenciadas entre os municípios, de acordo com o nível de eficiência destes. Além disso, deve-se levar em conta que grande parte dos municípios apresenta elasticidades de produção dos fatores terra e trabalho semelhantes aos dos municípios mais ineficientes. Assim, esses fatores deverão ser alocados de forma mais coerente, com vistas em reduzir as ineficiências na produção.

\section{Conclusões}

Este trabalho buscou apresentar uma proposta metodológica que indica alguns procedimentos analíticos para analisar a função de produção. Justifica-se tal proposta na medida em que existem diferentes níveis de eficiência produtiva nas unidades agrícolas analisadas, as quais podem comprometer a correta estimação da função de produção. Para testar a metodologia, foram utilizados dados referentes aos insumos e produtos agrícolas dos municípios da mesorregião Triângulo Mineiro/Alto Paranaíba, em Minas Gerais.

Os resultados foram coerentes, uma vez que foi possível identificar as diferenças nas elasticidades de produção e de escala das funções de produção média e de fronteira da agropecuária dos municípios, bem como discriminar os municípios relativamente ineficientes. Uma das principais conclusões é o fato de que existem diferenças significativas entre as funções de produções, indicando o viés que ocorre ao tomar decisões 
Antonio José Medina dos Santos Baptista, William Romell Benevídes de Ávila, João Eustáquio de Lima \& Adriano Provezano Gomes

baseadas somente nos parâmetros da função de produção média. Outra conclusão importante é o fato de que, pelos procedimentos adotados no estudo, podem-se evidenciar algumas características estruturais da tecnologia de produção, em determinada região.

Limitação importante do estudo é o fato de que a abordagem da programação de metas para estimar os parâmetros das funções de produção, além de não permitir testes de hipóteses nos parâmetros, as estimações das funções de produção de fronteira são sensíveis a observações discrepantes (outliers), podendo comprometer a estimação das funções fronteira. Não obstante essas limitações, os procedimentos apresentados neste estudo são de fundamental importância no auxílio à tomada de decisão e à formulação de políticas de promoção do setor agropecuário.

\section{Referências}

AIGNER, D. J., CHU, S. F. On estimating the industry production function. American Economic Review. 58:4 (September), 826-39. 1968.

DEBERTIN, D. L. Agricultural production economics. New York: Macmillan Publishing Company, 1998.366p.

FARE, R., GROSSKOPF, S., LOVELL, C.A.K. Production frontiers. Cambridge: Cambridge University, 1994. 295 p.

INSTITUTO BRASILEIRO DE GEOGRAFIA E ESTATÍSTICA IBGE. Banco de dados. [25/04/2005]. (http://www.sidra.ibge.gov.br/).

KUMBhaKAR, S. C., LOVELl, C. A. K., Stochastic frontier analysis. Cambridge: Cambridge University, 2000. 333 p.

SATO, K., Production functions and aggregation. Amsterdam: NorthHolland publishing company. 1975.313p. 
SCHMIDT, P. On the statistical estimation of parametric frontier production functions. Review of economics and statistics 58:2 (May) 238-39. 1976.

Abstract - The objective of this study was to analyze the average production functions and the frontiers production function to characterize the structure of the agricultural production technology. Using data of the agricultural census of 1995/96 regarding the mesorregião of the Triangulo Mineiro and Alto Paranaíba of the State of Minas Gerais the goal programming was used to calculate the parameters of the Cobb Douglas production functions. One of the main conclusions is the existence of significant differences in the level of productive efficiency and in the structure of the production technologies in this Mesorrregião, indicating the bias that is incurred when making decisions only based on the parameters of the average production function. Another important conclusion is the fact that the procedures adopted in this study, shows some evidence about the structural characteristics of the production technology offering better information for the decision makers.

Keywords: Production function; goal Programming; Efficiency; Agriculture. 